\title{
1 Yeast transformation efficiency is enhanced by \\ 2 TORC1- and eisosome-dependent signalling
}

3

4 Sheng-Chun Yu*, Florian Kuemmel, Maria-Nefeli Skoufou-Papoutsaki,

5 and Pietro D Spanu

6

7 Address: Department of Life Sciences, South Kensington Campus,

8 Imperial College London, London, SW7 2AZ, United Kingdom.

9

10

11

12

13

$14{ }^{*}$ Corresponding Author

15 Email: joescyu@gmail.com 


\section{ABSTRACT}

18 Transformation of baker's yeast (Saccharomyces cerevisiae) plays a key role in several experimental techniques, yet the molecular mechanisms underpinning transformation are still unclear. Addition of amino acids to the growth and transformation medium increases transformation efficiency. Here, we show that target of rapamycin complex 1 (TORC1) activated by amino acids enhances transformation via ubiquitin-mediated endocytosis. We created mutants of the TORC1 pathway, $\alpha$-arrestins, and eisosome-related genes. Our results demonstrate that the TORC1-Npr1-Art1/Rsp5 pathway regulates yeast transformation. Based on our previous study, activation of this pathway results in a 13-fold increase in transformation efficiency, or greater. Additionally, we suggest DNA is taken up by domains at the membrane compartment of Can1 (MCC) in the plasma membrane formed by eisosomes. Yeast studies on transformation could be used as a platform to understand the mechanism of DNA uptake in mammalian systems, which is clinically relevant to optimise gene therapy.

\section{INTRODUCTION}

Yeast transformation is the process by which exogenous DNA is introduced into the cell. It is a powerful tool of molecular biology research, for example in the yeast twohybrid $(\mathrm{Y} 2 \mathrm{H})$ system for detection of protein-protein interactions (Fields and Song 1989). Highly efficient protocols for chemical transformation have been established (Gietz 2015) but the molecular mechanisms underlying yeast transformation are not well understood. Several studies over the last four decades have investigated how DNA passes through the cell wall, through the plasma membrane (PM), and subsequently reaches the nucleus (Kawai, Hashimoto et al. 2010, Mitrikeski 2013). Foreign DNA is most likely to be engulfed via endocytic membrane invagination and several mutants involved in endocytosis show low transformation efficiencies (Kawai, Pham et al. 2004).

Ubiquitination of plasma membrane proteins can serve as an internalisation signal for endocytosis (Toret and Drubin 2006). In this way, the cell can downregulate receptors or transporters via transport to endosomes and lysosomal degradation 
47 (Ghaddar, Merhi et al. 2014). In yeast, this process is mediated by the Rsp5 ubiquitin

48 ligase that requires adaptor proteins for recruitment to the specific plasma membrane targets. Proteins that bind to Rsp5 and promote this function, include the arrestin-related trafficking adaptors (ARTs) such as Art1, Art3 and Bul1 (Yashiroda,

51 Oguchi et al. 1996, Lin, MacGurn et al.). Several amino acid transporters are

52 internalised by this form of endocytosis, including the general amino acid permease

53 (Gap1) and the arginine-specific permease (Can1). The latter are targets of Bul1 and

54 Art1, respectively (Ghaddar, Merhi et al. 2014). Phosphorylation of Art1 and Bul1/2

55 by the Npr1 kinase cause translocation of Art1 from the plasma membrane to the

56 Golgi apparatus and binding of Bul1 to the inhibitory 14-3-3 proteins. This prevents

57 internalisation of the plasma membrane permeases (MacGurn, Hsu et al. 2011,

58 O'Donnell 2012).

The target of rapamycin complex 1 (TORC1) is highly conserved among eukaryotes and functions as a master regulator of cell growth and metabolism through its own as well as downstream protein kinases. TORC1 activity depends on nutrient availability, and amino acids are potent stimulators in vivo (Conrad, Schothorst et al. 2014, Dokudovskaya and Rout 2015, González and Hall 2017). When active, TORC1 promotes ubiquitin-mediated endocytosis by inhibiting Npr1, which in turn is a negative regulator of $\alpha$-arrestins acting in endocytic cargo sorting. Npr1 phosphorylates Art1 and this prevents Art1 from associating with the PM. In this way, the target of rapamycin (TOR) pathway connects amino acid sensing with endocytosis (MacGurn, Hsu et al. 2011).

The downstream plasma membrane targets of Art1 include nutrient permeases that accumulate in the membrane compartment of Can1 (MCC). The membrane composition of MCC differs from other membrane compartments by its higher content of ergosterol (Grossmann, Opekarová et al. 2007). Eisosomes, first discovered in 1963 in early electron microscopy studies (Moor and Muhlethaler 1963), are cytosolic multi-protein complexes that form 50-300 nm deep furrow-like invaginations of the plasma membrane associated with the MCC region (Stradalova,

76 Stahlschmidt et al. 2009). Eisosomes act as a hub for various signalling pathways and they may play a role in endocytosis, although their exact function is not well 
78

understood (Walther, Brickner et al. 2006, Fröhlich, Moreira et al. 2009, Douglas and Konopka 2014).

Recently, we developed a yeast transformation protocol called SuccessAA (Yu, Dawson et al. 2016). Using this method, we found that adding nutrients to the transformation and competence reagents substantially increased transformation efficiencies. We speculated that the mechanism underlying this effect was due to the activation of the TORC1 complex, which in turn promotes DNA uptake via ubiquitinmediated endocytosis.

The aim of our study was to investigate the molecular mechanisms of yeast transformation and the events that lead to the increase in transformation efficiency by addition of nutrients. We found that mutations of endocytic components resulted in changes in transformation efficiencies supporting the hypothesis that TORC1 and ubiquitin-mediated endocytosis is key to yeast transformation. Moreover, the boosting effect was observed in several distinct strains, highlighting the potential for the general application of the SuccessAA protocol to budding yeast transformation.

\section{RESULTS}

\section{The addition of amino acids results in increased transformation} efficiency in different $S$. cerevisiae strains

The applicability of the SuccessAA protocol to different budding yeast strains was examined by transforming four $S$. cerevisiae strains with a $13.8 \mathrm{~kb}$ plasmid (Figure 1A) namely THY.AP4, BY4743, W303-1A and MaV203. In all cases, the transformation efficiency after the addition of nutrients was substantially higher than without nutrient addition. The observed increases in efficiency were 15-fold $(p=0.0079) ; 6$-fold $(p=0.0079)$ and 37-fold $(p=0.0079)$ for THY.AP4, BY4743 and W303-1A, respectively. We deduce that the genetic requirements for this effect are likely to be conserved in $S$. cerevisiae. Therefore, adding nutrients during yeast transformation may provide a generally applicable method to boost transformation efficiency for budding yeast. 
Yu et al. 5

A

Budding Yeast Strains

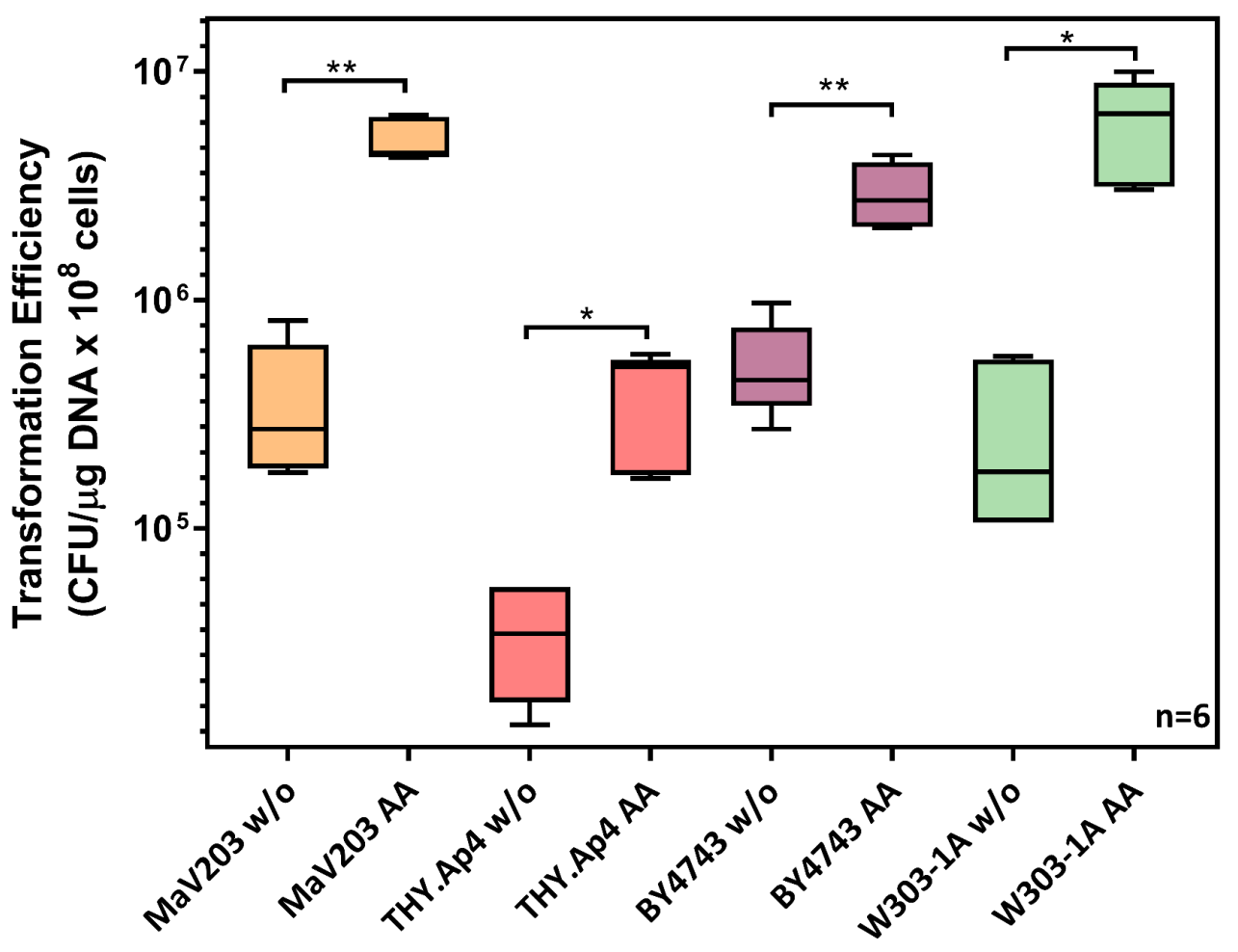

107

108

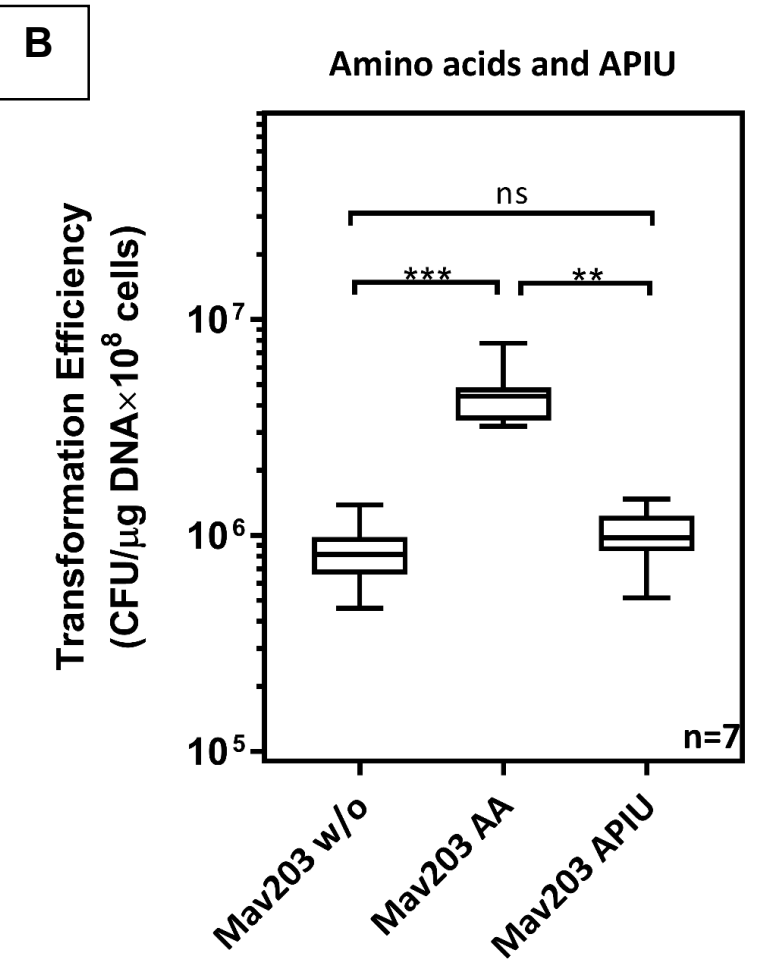


Figure 1. Amino acid addition results in higher transformation efficiency in different budding yeast strains.

111 (A) Four budding yeast strains, namely MaV203, THY.Ap4, BY4743, and W303-1A, were used to test the effect

112 of nutrient addition on boosting yeast transformation efficiency. Each one of the strains was transformed with

113 or without nutrient supplement (drop-out leucine) during the transformation process, followed by three-day

114 culture on 1x YPAD drop-out leucine plates and then the corresponding transformation efficiencies were

115 calculated. Statistical significance was assessed by using the Mann-Whitney test. Nutrient addition caused a

116 significant increase in transformation efficiency in all four yeast strains. Results are from six independent

117 biological replicates. (B) The MaV203 yeast strain was used to test if the boost effect was caused by either

118 amino acids or by four other nutrients, namely, adenine, p-aminobenzoic acid, inositol, and uracil (APIU).

119 MaV203 was transformed without nutrient addition, with amino acid addition (drop-out leucine), or with APIU

120 addition, followed by three-day culturing on 1x YPAD drop-out leucine plates. The corresponding efficiencies

121 were then assessed by the Kruskal-Wallis test, followed by Dunn's post-hoc test. Amino acid addition resulted

122 in significantly higher transformation efficiencies. Results are from seven independent biological replicates.

123

124 To determine which of the nutrients added in the SuccessAA protocol are necessary

125 for the boosting of competence, we tested the effect of a mixture of all amino acids

126 (AA) or Adenine, p-aminobenzoic acid, Inositol, and Uracil (APIU). The addition of

127 only APIU did not cause any significant change in the transformation efficiency

128 (Figure 1B) while amino acids caused the same increases seen in previous

129 experiments where a complex nutrient supplement was employed.

TORC1-regulated endocytosis is required for enhanced transformation

132 Previous studies demonstrated that amino acids in the growth medium activate the

133 TORC1 complex which in turn regulates ubiquitin-mediated endocytosis of nutrient

134 permeases via Npr1 and Art1 (MacGurn, Hsu et al. 2011). Here, we tested the

135 hypothesis that altering the rate of endocytosis by addition of amino acids leads to

136 increased DNA uptake. To achieve this, we created mutant strains lacking

137 components of the TORC1-dependent endocytic pathway (Figure 2), and analysed

138 the effect this had on transformation efficiency (Figure 3). We found that

139 transformation was no longer influenced by addition of amino acids to the medium

140 and transformation mix when either tco89 (the core subunit of TORC1, $\mathrm{p}=0.9619$ ) or

$141 \operatorname{art1}(p=0.9983)$ had been deleted. Conversely, the efficiency increases when $n p r 1$, 
142 a negative regulator of TORC1 mediated endocytosis, was missing. Note, this effect

143 was visible even in the absence of any additions to the medium (Tukey's multiple

144 comparisons test, $p=0.0021$ (wild type yeast without amino acid addition vs $n p r 1 \Delta$

145 without amino acid addition), but it was further enhanced when amino acids were

146 supplied ( $p<0.0001$ (wild type yeast with amino acid addition vs $\Delta n p r 1$ with amino

147 acid addition).

148 It has previously been reported that when TORC1 is inactivated, Npr1 stabilizes the 149 yeast plasma membrane general amino acid permease Gap1, by phosphorylating 150 alpha arrestin-like adaptors (Bul1/2); this leads to binding of 14-3-3 proteins and 151 cellular re-localization, which antagonises ubiquitin-mediated endocytosis (Merhi and 152 Andre 2012). Phosphorylation of $\alpha$-arrestins or arrestin-like adaptors (Art1, Art2, 153 Art3, Art5, Art6, and Bul1/2) increases in rapamycin-treated yeast cells

154 (lesmantavicius, Weinert et al. 2014). Art2 is an arrestin that does not regulate amino 155 acid induced endocytosis (Nikko and Pelham 2009) and Art5 targets a permease for 156 inositol, which is not involved in the transformation enhancing effect we observed. 157 Furthermore, the activity and the phosphorylation of Art3, but not Art6, are directly regulated by Npr1 (O'Donnell 2012).

a

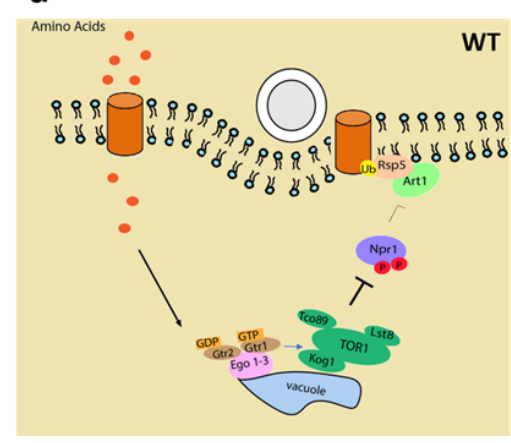

b

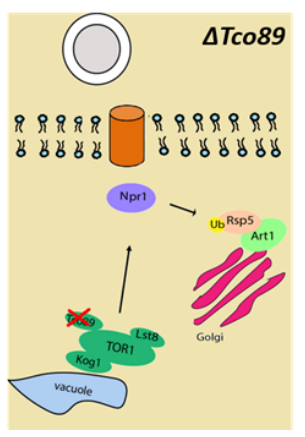

c

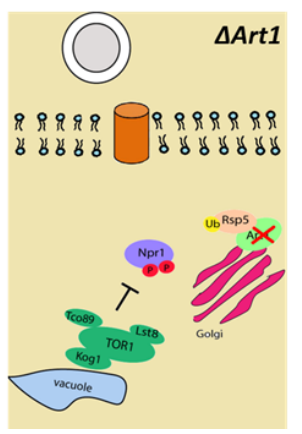

d

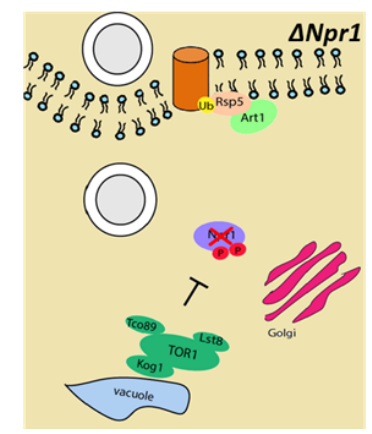

Figure 2. Illustration of the signalling pathways connecting perception of amino acids and endocytosis in wild-type, $\Delta$ tco89, $\Delta$ art1 and $\Delta$ npr1 cells.

(A) In wild type yeast, intracellular amino acids stimulate TORC1, via the Ego 1-3 complex. TORC1 in turn inhibits Npr1 by phosphorylation, allowing the alpha arrestin adaptor Art1 and ubiquitin ligase Rsp5 to be recruited in the plasma membrane and bind to amino acid transporters, leading to their ubiquitination, subsequent endocytosis and simultaneous plasmid DNA uptake. (B) When tco89 is deleted, TORC1 signalling is 
apparatus. Amino acid permeases endocytosis and simultaneous plasmid DNA uptake are hindered, as the amino acid permeases will not be ubiquitinated. (C) When art1 is deleted, Rsp5 cannot be recruited at the plasma membrane to bind to and ubiquitinate amino acid permeases Therefore, endocytosis of nutrient permeases and simultaneous plasmid DNA uptake are hindered. (D) When the negative regulator npr 1 is deleted, the inhibition on Art1's function in endocytosis is removed. Under this condition, binding of Art1/Rsp5 to amino acid permeases is increased and Art1/Rsp5 are continuously recruited to the plasma membrane, which, in turn, stimulates amino acid permeases and plasmid DNA invagination.

174 Here, we investigated the roles of Art1, Art3, Bul1 and the ubiquitin ligase Rsp5 in 175 facilitating the increase in transformation by targeted gene deletion and a complementation. The transformation efficiencies of $\Delta a r t 1, \Delta b u l 1$, and $\Delta$ art 3 cells were compared to that of wild type $S$. cerevisiae (Figure 3C). The median transformation efficiencies of $\Delta a r t 1, \Delta a r t 3$ and $\Delta b u l 1$ without amino acid addition was not significantly higher than those of wild-type yeast without amino acid addition (Tukey's multiple comparisons test, $\mathrm{p}=0.5640$ (wild-type vs $\Delta$ art1), $\mathrm{p}=0.9195$ (wildtype vs $\Delta$ art3), $p=0.9908$ (wild-type vs $\Delta b u l 1)$ ). When amino acids were added to the $\Delta$ art3 and $\Delta b u l 1$ strains, transformation efficiencies were substantially higher for both $\Delta a r t 3$ and $\Delta b u l 1$ (up to about 20 -fold; Tukey's multiple comparisons test, $p<0.0001$ ).

184 In contrast, as seen before, there was no boosting effect in the $\Delta$ art1 mutant 185 (Tukey's multiple comparisons test, $\mathrm{p}=0.9367$ ). We deduce from this that TORC1Npr1-Art1 signalling is specifically required for the boost in transformation efficiency induced by addition of amino acids to the media.

To test this hypothesis further, and to elucidate the role of the Rsp5, we carried out complementation of these mutants by 1) by the wild-type art1 gene (pRS426-Ldb19) and a mutant art1 gene from which the Rsp5-binding domain is deleted (pRS426Ldb19PPxY-less) (Figure 2D). We found that $\Delta$ art1 was effectively rescued by the wild type gene: addition of amino acids significantly increased (over 24-fold; Tukey's multiple comparisons test, $p<0.0001)$. Conversely, there was no significant difference when $\Delta$ art 1 was complemented by the gene lacking the Rsp5-binding domain (Tukey's multiple comparisons test, $p=0.6103$ ). In summary, we found that the enhancement of transformation in response to addition of amino acids is mediated by TORC1-Npr1-Art1/Rsp5 (coloured) rather than other TORC1-Npr1arrestins/Rsp5 routes (grey) (Figure 3D). Moreover, Rsp5 binding to Art1 is essential for this, which indicates that Rsp5-mediated mono-ubiquitination of plasma 
200 membrane cargo followed by ubiquitin-mediated endocytosis are necessary for 201 enhanced yeast transformation.

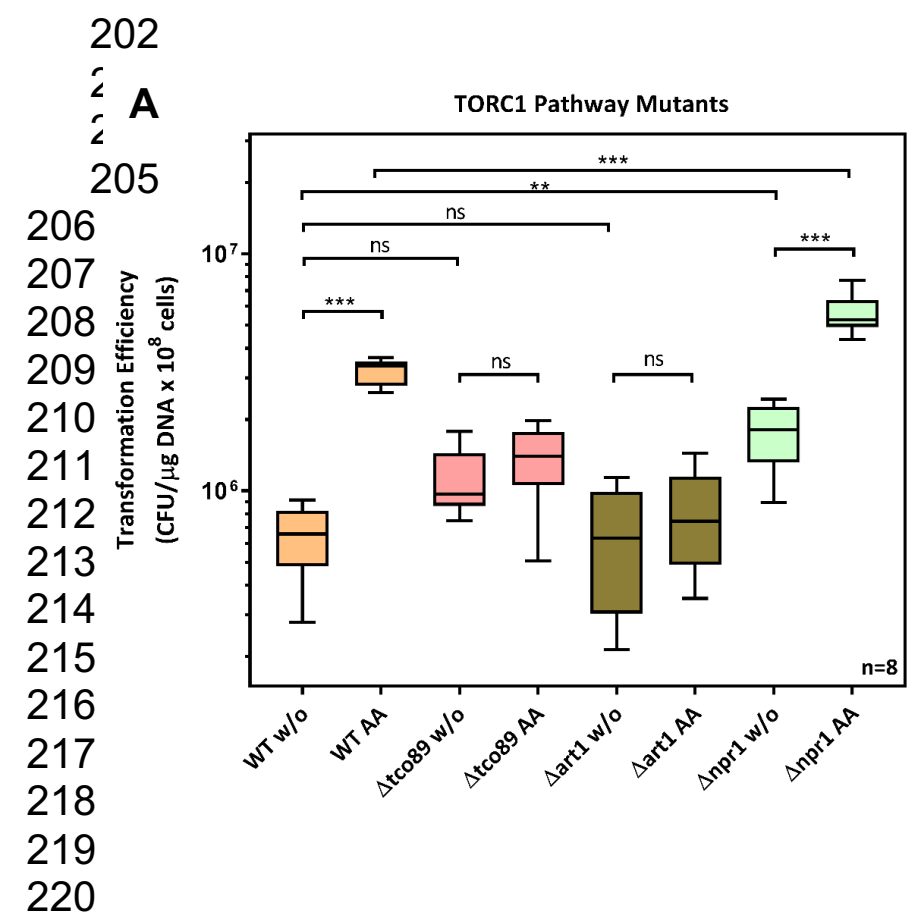

B

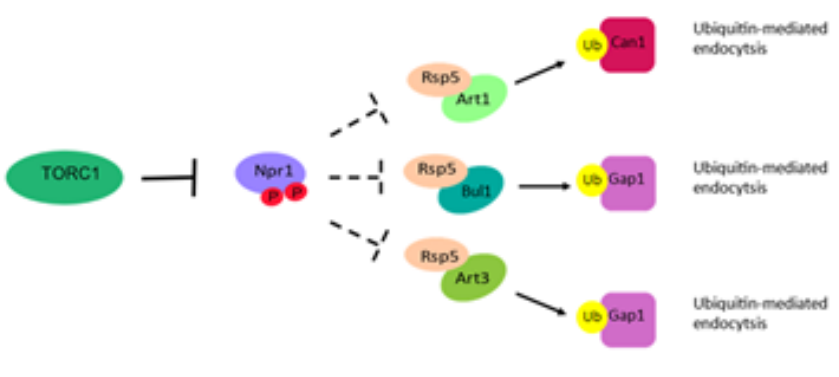

221

222

C

The role of Arts on yeast transformation

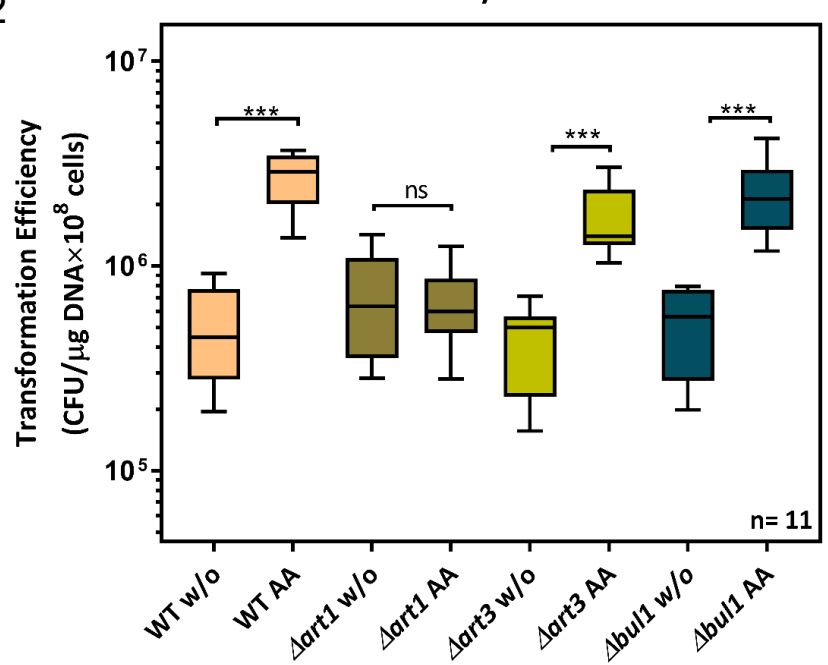

E

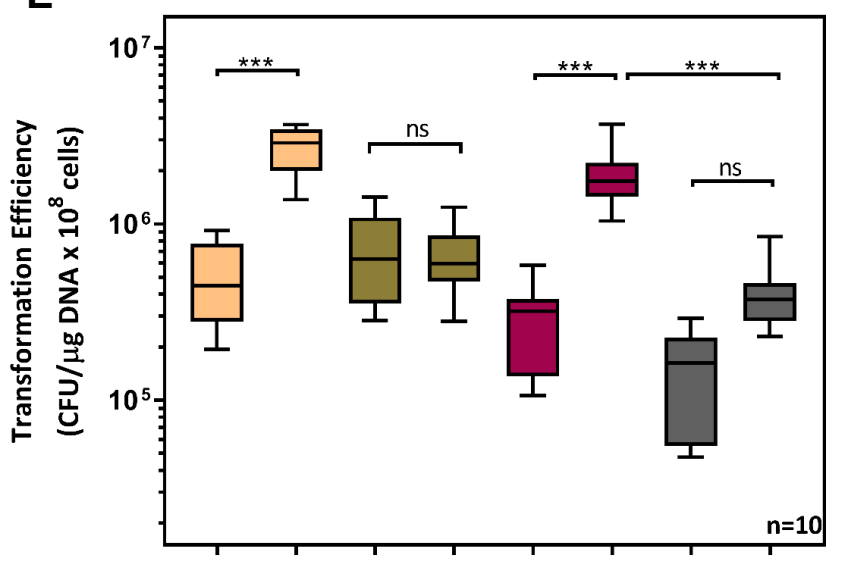

D

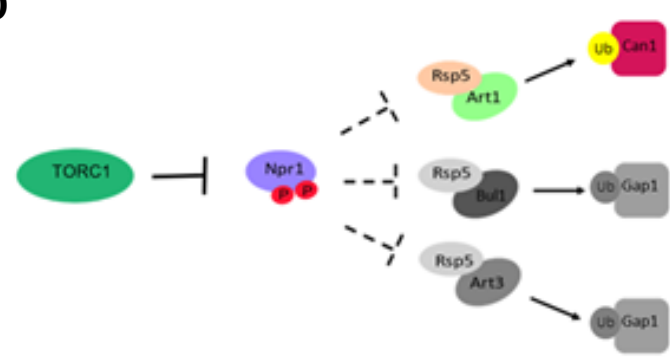
endocytsis

Ubiquitin-mediuted Ubiquitin-m
endocytsis 
Figure 3. The TORC1 signalling pathway is involved in yeast transformation.

227 (A) Three genes involved in TORC-1 signally were deleted from S. cerevisiae MaV203: the three strains

228 generated were $\Delta t c 089, \Delta a r t 1$, and $\Delta n p r 1$. Wild-type yeast (MaV203) and the mutant strains were

229 transformed either with or without amino acid addition. The corresponding efficiencies were assessed by

230 Tukey's multiple comparisons test. The boosting effect was absent when either tco89 or art1 was deleted

231 while transformation was enhanced when $n p r 1$ was deleted. Results are from eight independent biological

232 replicates. (B) The cartoon shows the pathways from TORC-1 to Npr1's downstream targets, Art1, Art3, and

233 Bul1. When TORC1 is active, the Npr1 kinase is inhibited, which allows Art1/Rsp5 binding to amino acid

234 permease Can1, followed by Can1 invagination. When npr1 is inhibited, it also allows Art3/Rsp5 and Bul1/Rsp5

235 acting in ubiquitin-dependent cargo-selection of the general amino acid permease Gap1. (C) The requirement

236 of different arrestins for transformability was tested. Three MaV203 mutants were generated, namely, $\Delta a r t 1$,

$237 \Delta a r t 3, \Delta b u l 1$, and these mutants were transformed either with or without amino acid addition, followed by

238 three-day culturing on 1x YPAD drop-out leucine and tryptophan. The corresponding transformation

239 efficiencies were assessed by Tukey's multiple comparisons test. Boosting was abolished only when Art1 was

240 deleted suggesting that the effect is mediated by TORC1-Npr1-Art1 signalling route. Results are from eleven

241 independent biological replicates. (D) The boosting effect is mediated by TORC1-Npr1-Art1/Rsp5 signalling (in

242 colour) while both TORC1-Npr1-Art3/Rsp5 signalling and TORC1-Npr1-Bul1/Rsp5 signalling are not involved in

243 boosting (in grey colour). (E) The Art1-PPxY-motif is required for effective plasmid DNA uptake. MaV203,

$244 \Delta$ art1, $\Delta$ art1 carrying pRS426-Ldb19 (art1+), and $\Delta$ art1 carrying pRS426-Ldb19PPxY-less (the PPxY motif

245 required for Rsp5 binding was deleted from Art1) were transformed either with or without amino acid

246 addition. After the transformation, the cells were cultured on 1x YPAD selection plates for three-days, followed

247 by assessing the efficiencies by Tukey's multiple comparisons test. The results demonstrated that

248 complementation of Art1 in $\Delta a r t 1$ restored boosting. However, the effect was not observed when Art1 was

249 expressed without the Rsp5 binding ability. Results are from ten independent biological replicates. 


\section{Seg1 is required for high-efficiency yeast transformation}

252 The integrity of eisosomes is known to affect the efficacy of endocytosis (Murphy,

253 Boxberger et al. 2011). Here, we investigated the effect of MCC/eisosome formation

254 on yeast transformation by deletion of seg1 (known to impair the formation of

255 eisosomes (Moreira, Schuck et al. 2012) and by deletion of ypk1 (a kinase involved

256 in eisosome formation (Luo, Gruhler et al. 2008)) (Figure 4). We found that removing

257 seg1 or ypk1 resulted in no amino-acid induced increase in transformation efficiency

258 ( $\Delta$ seg1 without amino acid addition vs $\Delta$ seg 1 with amino acid addition; $p=0.9873$;

$259 \Delta y p k 1$ without amino acid addition vs $\Delta y p k 1$ with amino acid addition, $p=0.9976)$. It

260 is noteworthy that although the boosting effect on both $\Delta$ seg 1 or $\Delta y p k 1$ disappeared,

261 there were evident differences in the basal transformation efficiencies in the absence

262 of added amino acids to the media (wild-type vs $\Delta$ seg1: $p=0.0249$; wild-type vs

$263 \Delta y p k 1: \mathrm{p}=0.0118)$.

\section{The role of eisosomes on yeast transformation}

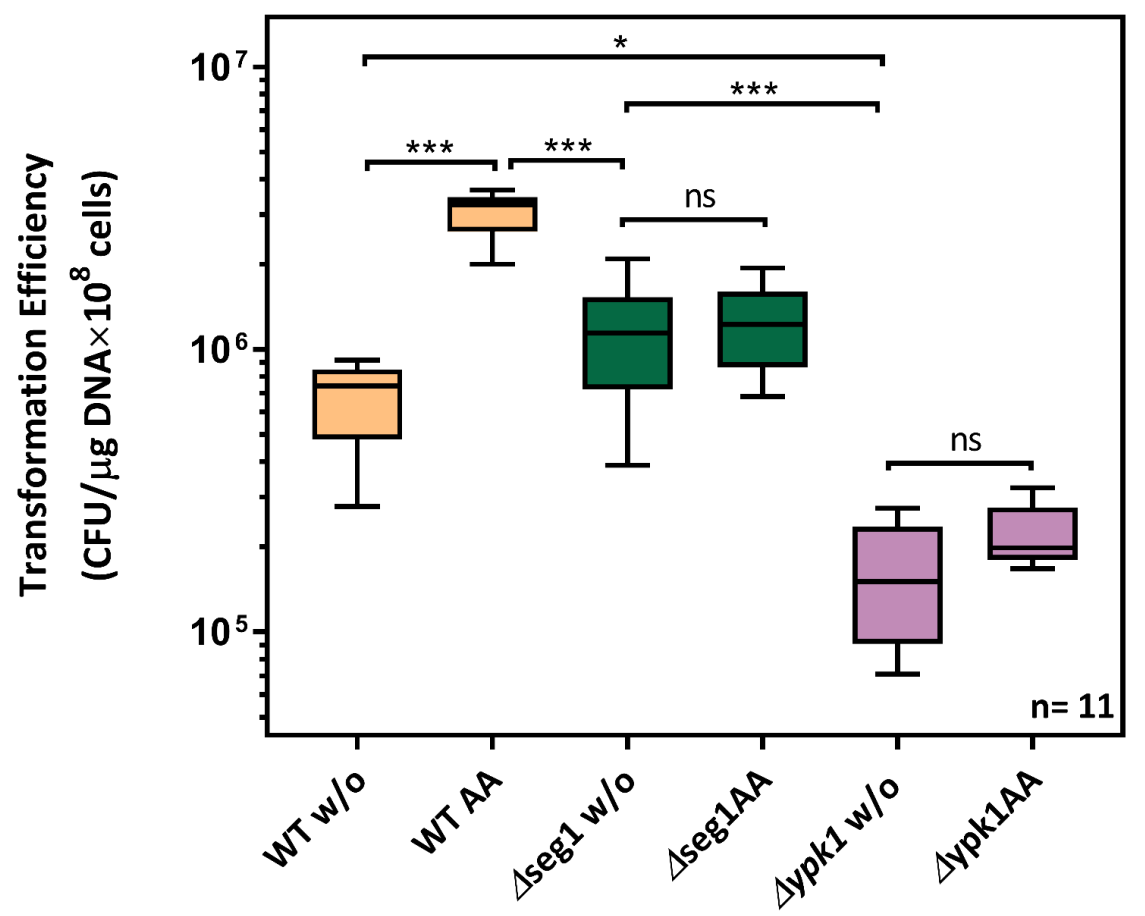

Figure 4. Eisosomes are required for increased transformation efficiency in yeast. $\Delta$ seg 1 and $\Delta y p k 1$ strains generated by deletion of the respective genes were transformed either with or without amino acid addition to the media. The corresponding transformation efficiencies were analysed by 
$\Delta s e g 1$ and $\Delta y p k 1$, suggesting that functional eisosome formation and signalling are required for highly efficient yeast transformation. Results are representative of 11 independent biological

271 replicates.

\section{Discussion}

273 Yeast transformation has been described for almost 40 years (Hinnen, Hicks et al.

274 1978) and is a cornerstone of many fundamental methods in genetics, cell biology as 275 well as practical biotechnological applications. It is therefore surprising there are only 276 few mechanistic explanations of the processes underpinning this key technique 277 although several have been proposed (Beggs 1978). One model suggests that 278 foreign DNA is engulfed via endocytic membrane invagination; this is supported by 279 the observation that several low transformability phenotypes are caused by mutation 280 of genes involved in endocytosis (Kawai, Pham et al. 2004). Here, we tested the extent to which targeted deletions of single endocytic genes affect competence, and we observed how the changes to nutrients in the growth and the transformation media affected transformation efficiency. We found that adding amino acids boosted competence in all four strains of $S$. cerevisiae tested, demonstrating that this phenomenon is likely to be generally applicable in budding yeast. We propose a model to summarise the processes described here (Figure 5).

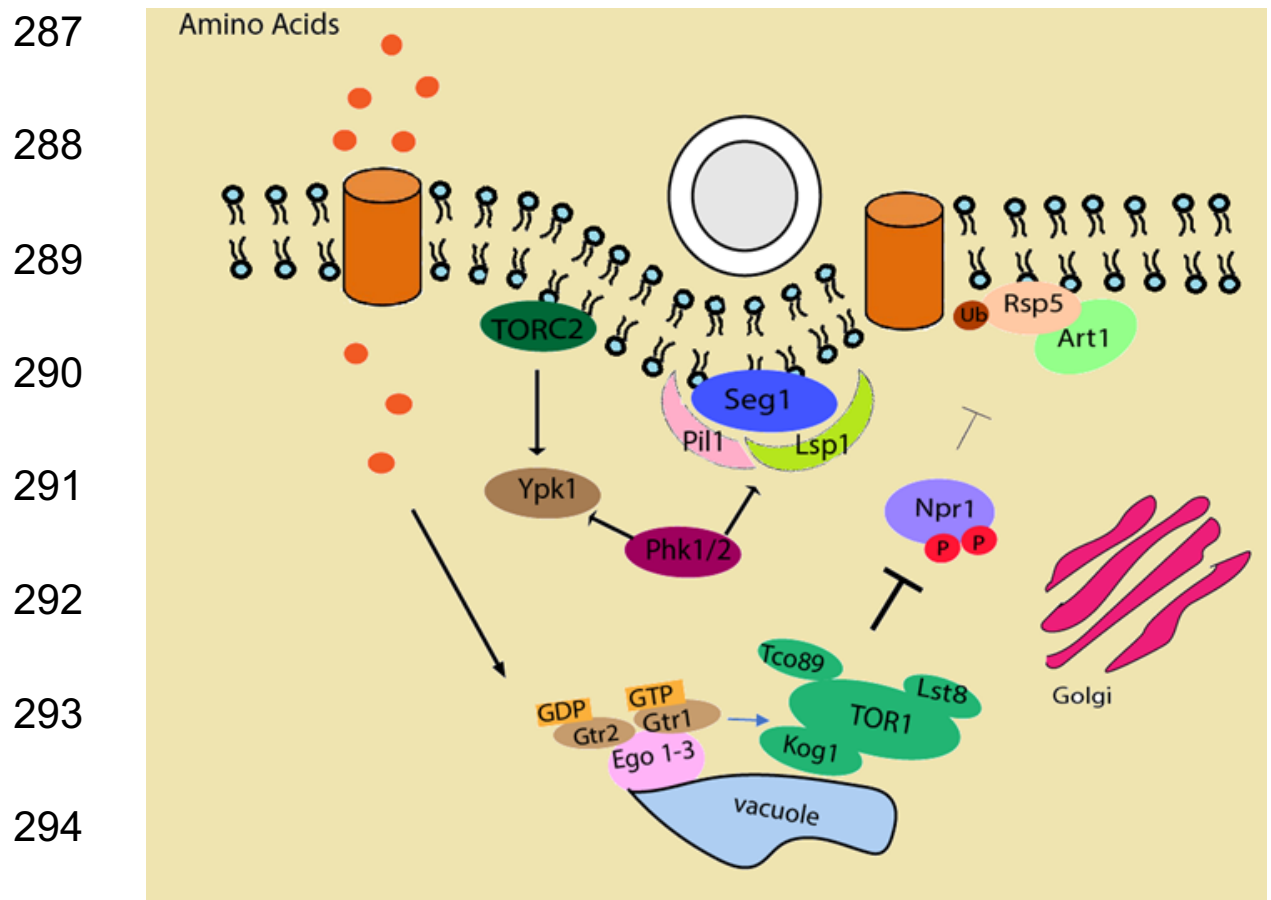


Figure 5. Proposed model of TORC1-regulated DNA uptake in yeast and the involvement of eisosomes.

Amino acids are transported into the cell cytoplasm via amino acid permeases, where they stimulate TORC1.

Activated TORC1 inhibits the Npr1 which, in turn, facilitates Art1/Rsp5-dependent cargo selection and

304 The tco89 gene encodes a subunit of TORC1. We observed that in $\Delta t c 089$ cells

305 there was never any boosting effect in response to nutrient stimuli. However, deletion of tco89 did not lead to changes in basal competence, but only affected the boost induced by amino acids in the media. Therefore, whileTORC1 is necessary for the regulation of the amino acid-induced effect, there are also other pathways underpinning DNA uptake. Plasma membrane permeases such as Can1 are under the control of TORC1, but are also internalized in response to their substrate,

311 independently of TORC1 signalling (Opekarova, Caspari et al. 1998, Ghaddar, Merhi 312 et al. 2014). Our results imply that, in contrast to TORC1-regulated internalization, 313 the influx-stimulated internalization of PM permeases does not contribute to efficient 314 DNA uptake or subsequent delivery to the nucleus.

In contrast, the basal competence of $\Delta n p r 1$ cells was higher than wild type. This was attributed to abolition of the of $\alpha$-arrestins' inhibition of these endocytic processes

317 (MacGurn, Hsu et al. 2011). The boost of transformation observed in $\Delta n p r 1$ cells 318 treated with amino acids reveals there are other regulators of arrestins under the 319 control of TORC1, in addition to Npr1.

We discovered that a functional Art1 was indispensable for high transformability in response to amino acids. In experiments where we complemented the art1 deletion,

323 we also observed that the Rsp5-binding domain of Art1 was required for the boosting 324 effect. Because of this, we propose that ubiquitin-mediated cargo sorting is involved 325 in efficient DNA uptake in vivo. 
While Art1 was needed for high transformability in response to amino acids, deletion of other arrestins that act in a similar manner (Bul1, Art3) had no effect. This is important as both Bul1 and Art3 are under the control of Npr1 and are involved in endocytosis (O'Donnell, Apffel et al. 2010, O'Donnell 2012). Thus, the TORC1 Npr1-Art1 pathway is specifically responsible for the effect on competence investigated here. This might be explained by the observation that the three arrestins we tested have different targets localised to different domains of the plasmamembrane. For example, Art1 is involved in Can1 endocytosis, whereas Bul1 and Art3 function in Gap1 down-regulation and recycling (Helliwell, Losko et al. 2001, Lin, MacGurn et al. 2008, O'Donnell, Apffel et al. 2010). In the plasma membrane, whilst Gap1 is uniformly distributed (Lauwers, Grossmann et al. 2007), there are specific compartments containing Can1 (MCC) (Nikko and Pelham 2009): therefore, these results hinted that MCC may be important domain in transformation competence.

341 Eisosomes are cytosolic multi-protein complexes that form 50-300 nm deep 342 invaginations of the plasma membrane associated with the MCC domain 343 (Stradalova, Stahlschmidt et al. 2009). The membrane composition of the MCC 344 differs from other yeast membrane compartments because it contains more 345 ergosterol (Grossmann, Opekarová et al. 2007). Eisosomes act as a hub for various signalling pathways and may play a role in endocytosis (Walther, Brickner et al. 2006, Fröhlich, Moreira et al. 2009, Douglas and Konopka 2014). Research on endocytic activity associated to eisosomes has led to several intensely debated results (Grossmann, Malinsky et al. 2008, Vangelatos, Roumelioti et al. 2010, Brach, 350 Specht et al. 2011, Murphy, Boxberger et al. 2011, Seger, Rischatsch et al. 2011, 351 Athanasopoulos, Boleti et al. 2013). Eisosomes require seg1 for stability (Moreira, 352 Schuck et al. 2012).

353 Our finding that mutant strains lacking seg1 do not show high transformability in response to an amino acid stimulus (Figure 2E), is indirect support for claims that eisosomes either mark sites of endocytosis or positively regulate endocytosis (Walther, Brickner et al. 2006, Murphy, Boxberger et al. 2011). Importantly, although 
transformation is unlikely to be restricted to the boosting effect, because a subset of eisosomes still forms in $\Delta$ seg 1 cells (Moreira, Schuck et al. 2012).

360 One of the functions of YPK1 kinase is to control eisosome formation (Luo, Gruhler et al. 2008). Deletion of YPK1 led to the lowest transformation efficiencies out of all mutant strains we tested in this study. However, further work is needed to clarify the exact role of Ypk1 in DNA uptake because: Ypk1 also regulates at least one $\alpha-$ arrestin (Alvaro, Aindow et al. 2016), it impinges on actin dynamics (Niles and Powers 2014), and it is involved in the heat stress response (Sun, Miao et al. 2012). The hypotheses that eisosomes mark sites of endocytosis and that transformation is facilitated by endocytosis have one point in common: in both cases these types of endocytosis differ from well-studied endocytic pathways, such as clathrin-mediated endocytosis that originates at actin patches (Kawai, Pham et al. 2004, Ziółkowska,

370 Christiano et al. 2012).

371 Alternative endocytic pathways in yeast are not as well-studied as in mammalian 372 cells. New insights emerged in recent years, for example the $\alpha$-arrestins Art1 and

373 Bul1 can lead to endocytic downregulation of transmembrane transporters in a 374 clathrin- and ubiquitin-independent manner, relying on Rho1 (Prosser, Drivas et al. 375 2011, Prosser, Pannunzio et al. 2015). Indeed, as stated above, we found that the 376 Rsp5-binding domain in Art1 was required to observe high transformation efficiency.

377 This implies that ubiquitination of cargo proteins does at least partially contribute to 378 subsequent DNA uptake. Whether endocytic DNA uptake relies on clathrin-coated 379 vesicles and ubiquitination as a cargo signal per se, remains to be seen.

380 Nevertheless, we propose that an eisosome-mediated pathway is the main route for 381 efficient DNA delivery into the yeast cell.

\section{Outlook}

383 A complete mechanistic description of the genetic requirements and endocytotic mechanism for nucleic acid uptake is immensely important not just for understanding yeast transformation but also for further progress in various fields like gene therapy in humans, understanding RNA trafficking and improving RNA interference

387 technologies. 
388

389

390

391

392

393

394

395

396

397

398

399

400

401

402

403

404

405

406

407

408

409

410

411

412

413

414

415

416

417

418

It will be exciting to see whether the concept of achieving high transformation efficiencies in yeast by stimulation of TORC1 can be applied to the mammalian mTORC system as well. By highlighting the importance of the metabolic state of the cell, this study opens up new practical possibilities for the improvement of transformation efficiencies, by fine-tuning the nutrient composition in the transformation reagent.

\section{MATERIALS AND METHODS}

\section{S. cerevisiae strains, plasmids, reagents and equipment}

This study includes an evaluation of transformation efficiencies of four S. cerevisiae strains, under different nutrient conditions. Namely, the yeast strains MaV203 (from ProQuest $^{\mathrm{TM}}$ Two-Hybrid system (PQ10001-01, Thermo Fisher Scientific), W303-1A, BY-4743, and THY.AP4 (kindly provided by Bjorn Sabelleck, RWTH Aachen University). The MaV203 strain was used to generate seven mutant strains. These included $\Delta a r t 1, \Delta a r t 3, \Delta b u l 1, \Delta n p r 1, \Delta s e g 1, \Delta t c 089$, and $\Delta y p k 1$. Four plasmids, namely, pDEST22 (PQ1000101, Thermo Fisher Scientific), pDEST32-TaRNR8-p12L (generated by Dr Sheng-Chun Yu), pRS426-Ldb19, and pRS426-Ldb19 ${ }^{\text {PPxY-less }}$ the last two plasmids kindly provided by Allyson F. O'Donnell, Duquesne University, PA

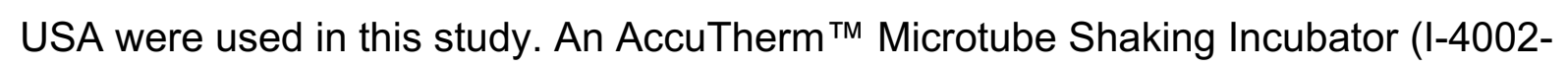
HCS, Labnet International, Inc.) was used for the heat-shock process in yeast transformation. We used the following reagents in this study: Yeast extract (Y1625250G, Sigma-Aldrich), Peptone (P5905-1KG, Sigma-Aldrich), Adenine hemisulfate salt (A3159-100G, Sigma-Aldrich), D-(+)-Glucose (G7021-1KG, Sigma-Aldrich), yeast nitrogen base without amino acids (Y0626-250G, Sigma-Aldrich), yeast synthetic drop-out medium supplements (Y2001-20G, Sigma Aldrich), L-histidine monohydrochloride monohydrate (53370-100G, Sigma-Aldrich), L-tryptophan (T8941-25G, Sigma-Aldrich), uracil (U1128-25G, Sigma-Aldrich), D-sorbitol (S38891KG, Sigma-Aldrich), Poly(ethylene glycol) BioUltra, 1000 (PEG1000) (81188- 250G, Sigma-Aldrich), LiAc (6108-17-4, Alfa Aesar), Deoxyribonucleic acid sodium salt from salmon testes (ss-DNA) (D1626-5G, Sigma-Aldrich), Bicine (B3876-100G, Sigma-Aldrich), ethylene glycol(324558-100ML, Sigma-Aldrich), dimethyl sulfoxide (DMSO) (D2650-5 × 5ML, Sigma-Aldrich), Water Molecular Biology Reagent 
419 (W4502, Sigma-Aldrich), Ultra-Pure ${ }^{\text {TM }}$ Agarose (16500500, Thermo Fisher

420 Scientific), SYBR® Safe DNA Gel Stain (S33102, ThermoFischer Scientific),

421 GeneRuler 1kb Plus DNA ladder (SM0311, ThermoFischer Scientific), Zymolyase

422 from Easy Yeast Plasmid Isolation Kit (630467, Clontech), GoTaq® G2 DNA

423 Polymerase and 5X Colorless GoTaq® Reaction Buffer (M7841, Promega) and

424 dNTP mix (R0191, ThermoFischer Scientific).

\section{S. cerevisiae transformation}

426

S. cerevisiae transformations were performed using the SuccessAA protocol ( $\mathrm{Yu}$,

427 Dawson et al. 2016), an adaptation of the LiAc/SS carrier DNA/PEG method (Gietz

428 2015) with the addition of amino acids in the transformation mix. The concentration

429 of amino acids used was $1.25 \mathrm{X}$ the concentration of amino acids found in Synthetic

430 Complete (SC) medium. Briefly, $0.25 \mu \mathrm{g}$ endotoxin-free pDEST32-TaRNR8-p12L

431 plasmid (13.8kb) was added into $50 \mu \mathrm{l} \mathrm{MaV203} \mathrm{competent} \mathrm{cells,} \mathrm{followed} \mathrm{by} \mathrm{adding}$

$432500 \mu$ l transformation mix solution, containing 36\% (w/v), PEG 1000, 0.1 M LiAc, 0.2

$433 \mathrm{mg} / \mathrm{ml} \mathrm{ss}-\mathrm{DNA}, 0.2 \mathrm{M}$ Bicine- $\mathrm{NaOH}(\mathrm{pH}=8.35)$, and 1.25x amino acid mix solution.

434 The plasmid DNA was mixed to the competent cells in the transformation mix

435 solution, and the yeast cells were then heat-shocked in the microtube shaking

436 Incubator at $37^{\circ} \mathrm{C}$ for 30 minutes. The transformation mixtures were shaken at the

437 start and after 15 minutes at 1500 rpm for 5 seconds; at the end of the incubation,

438 the samples were shaken for 30 seconds. After the heat shock, $50 \mu$ transformation

439 mixtures containing wild type MaV203 yeast cells or different mutated MaV203 yeast

440 strains were plated on suitable synthetic "drop-out" plates, and cultured for three

441 days at $30^{\circ} \mathrm{C}$. The numbers of colony forming units (CFU) were counted, and

442 transformation efficiencies $(E)$ were calculated with the following formula:

$$
E=\frac{C F U}{\mu g D N A \times 10^{8} \text { cells }}
$$

446 The current study generated seven yeast mutants to investigate the potential

447 molecular mechanisms underlying yeast transformation. Targeted gene deletion 
mutagenesis (gene "knock-out") mediated by homologous recombination reaction was used to mutate the following genes in MaV203: art1, art3, bul1, npr1, seg1, tco89, and ypk1. Mutagenesis primers were designed so that the gene of interest would be replaced by TRP1, which served as an auxotrophic selection marker carried by the pDEST22 plasmid. Primer sequences are shown in Supplementary Table 1. All the sequences of the forward primers were 74 bases whereas the first 50 bases were identical to the first 50 bases of the target gene, followed by the reverse and complementary 24-base sequence (6431bp to 6454bp on pDEST22) adjacent to the ARS/CEN locus in the pDEST22 plasmid. Similarly, the sequences of the reverse primers were 74 bases whereas the first 50 bases were identical to the last 50 bases of the target gene, followed by the reverse and complementary 24-base sequence (5143bp to $5166 \mathrm{bp}$ ) which is adjacent to the $\mathrm{f} 1$ origin in the pDEST22 plasmid. The plasmid pDEST22, carrying TRP1, was used as a PCR template. The PCR thermal cycled we used was: initial denaturation at $95^{\circ} \mathrm{C}$ for 3 minutes, followed by 40 cycles of denaturation at $95^{\circ} \mathrm{C}$ for 30 seconds, annealing at $45^{\circ} \mathrm{C}$ for 30 seconds, and extension at $72^{\circ} \mathrm{C}$ for 2 minutes and then the final extension at $72^{\circ} \mathrm{C}$ for 7 minutes. The PCR products were examined by gel electrophoresis. Once the PCR products exactly matched the predicted size, the PCR product was purified using QIAquick PCR Purification Kit. The gene specific PCR products were used to transform $S$. cerevisiae MaV203 as as described above. A $100 \mu$ l aliquot of the transformation mixture was plated on synthetic complete "drop out" tryptophan plates, followed by culturing the plates at $30^{\circ} \mathrm{C}$ for three days. Potentially mutated MaV203 yeast colonies were analysed using a modified version of yeast colony PCR protocol published in Molecular Cloning: A Laboratory Manual (Green 2012). In brief, at least ten colonies on each plate were randomly selected and approximately $1 / 10^{\text {th }}$ of each colony was carefully transferred to each sterile PCR tube, containing $5 \mu$ zymolyase

474 solution (from Easy Yeast Plasmid Isolation Kit). The PCR tubes with yeast-

475 zymolyase mix were then incubated for 30 minutes at $37^{\circ} \mathrm{C}$, followed by incubating

476 for 10 minutes at $95^{\circ} \mathrm{C}$ to inactivate zymolyase. After zymolyase inactivation, the 477 yeast-zymolyase mix was diluted by addition of $95 \mu \mathrm{l}$ molecular biology grade 478 endotoxin-free water and then yeast colony PCR was performed. The yeast colony 479 PCR program was as follows: initial denaturation at $95^{\circ} \mathrm{C}$ for 5 minutes, followed by 48040 cycles of denaturation at $95^{\circ} \mathrm{C}$ for 1 minute, annealing at $53^{\circ} \mathrm{C}$ for 1 minute, and 481 extension at $72^{\circ} \mathrm{C}$ for 2 minutes and then the final extension at $72^{\circ} \mathrm{C}$ for 7 minutes. 
482 When the colony PCR finished, $5 \mu$ PCR reactions were analysed on a $1.5 \%(\mathrm{w} / \mathrm{v})$

483 agarose/TBE gel for 45 minutes at $10 \mathrm{~V} / \mathrm{cm}$. Successful transformants were identified 484 based on the predicted PCR product length, for that primers of the adjacent down485 and upstream region of the target gene where designed. Reverse primers binding to 486 TRP1 were used in a separate PCR reaction for an additional verification.

\section{Evaluation of transformation efficiencies}

488 Once the mutants were confirmed by yeast colony PCR, the mutants were cultured 489 on 1x YPAD "drop-out Tryptophan" plates for three days, followed by growth in the same 1xYPAD "drop-out" medium overnight. Frozen yeast mutant competent cells were then prepared, transformed, and cultured on 1XYPAD "drop-out leucine and

492 tryptophan" plates using the SuccessAA protocol (Yu, Dawson et al. 2016). Mutant

493 transformation efficiencies were measured and compared to the efficiency of wild 494 type MaV203 yeast cells.

496 The collected data was not always normally distributed so non-parametric tests were 497 used, where appropriate. In the evaluation of the transformation efficiency of different 498 budding yeast strains, there was no inter-group comparison, only two groups were 499 compared at a time (same yeast strain, with the addition of amino acids or without).

500 Thus, the Mann-Whitney test were used to assess statistical significance. For 501 assessment of APIU's effect on transformation efficiency, there were three groups to 502 be compared so a Kruskal Wallis test, followed by Dunn's post-hoc test was used. In 503 the rest of the experiments, there was an interaction between the two factors tested, 504 the addition of amino acids and different mutant yeast strains. For this reason, two505 way ANOVA was used to assess statistical significance, followed by Tukey's multiple comparisons test. Statistical analysis was performed in GraphPad Prism 7.03.

\section{Competing interests}

509 The authors declare no competing financial interests. 


\section{Contributions}

511 S.-C.Y. conceived the original idea of this study. S.-C.Y., F.K. and N.S.P. designed 512 and carried out the experiments, analysed the data and drafted the paper. P.D.S.

513 supervised and advised the work and edited the manuscript.

\section{Acknowledgements}

515 We thank Mark Isalan (Imperial College London) for invaluable advice on yeast 516 mutagenesis Allyson O'Donnell (Duquesne University, PA USA) who kindly provided 517 and the pRS426-Ldb19, and pRS426-Ldb19 ${ }^{\text {PPxY-less }}$ plasmids.

\section{References}

519 Alvaro, C. G., A. Aindow and J. Thorner (2016). "Differential phosphorylation provides a 520 switch to ontrol how alpha-arrestin Rod1 down-regulates mating pheromone response in 521 Saccharomyces cerevisiae." Genetics 203(1): 299-317.

522 Athanasopoulos, A., H. Boleti, C. Scazzocchio and V. Sophianopoulou (2013). "Eisosome 523 distribution and localization in the meiotic progeny of Aspergillus nidulans." Fungal Genet 524 Biol 53: 84-96.

525 Beggs, J. D. (1978). "Transformation of yeast by a replicating hybrid plasmid." Nature 526 275(5676): 104-109.

527 Brach, T., T. Specht and M. Kaksonen (2011). "Reassessment of the role of plasma 528 membrane domains in the regulation of vesicular traffic in yeast." J Cell Sci 124(Pt 3): 328529337.

530 Conrad, M., J. Schothorst, H. N. Kankipati, G. Van Zeebroeck, M. Rubio-Texeira and J. M. 531 Thevelein (2014). "Nutrient sensing and signaling in the yeast Saccharomyces cerevisiae." 532 FEMS Microbiol Rev 38(2): 254-299.

533 Dokudovskaya, S. and M. P. Rout (2015). "SEA you later alli-GATOR--a dynamic regulator of 534 the TORC1 stress response pathway." J Cell Sci 128(12): 2219-2228.

535 Douglas, L. M. and J. B. Konopka (2014). "Fungal membrane organization: the eisosome 536 concept." Annu Rev Microbiol 68: 377-393.

537 Fields, S. and O. Song (1989). "A novel genetic system to detect protein-protein 538 interactions." Nature 340: 245.

539 Fröhlich, F., K. Moreira, P. S. Aguilar, N. C. Hubner, M. Mann, P. Walter and T. C. Walther 540 (2009). "A genome-wide screen for genes affecting eisosomes reveals Nce102 function in 541 sphingolipid signaling." The Journal of Cell Biology 185(7): 1227.

542 Ghaddar, K., A. Merhi, E. Saliba, E. M. Krammer, M. Prévost and B. André (2014). "Substrate543 induced ubiquitylation and endocytosis of yeast amino acid permeases." Molecular and 544 Cellular Biology 34(24): 4447-4463. 
545 Gietz, R. D. (2015). High Efficiency DNA Transformation of Saccharomyces cerevisiae with

546 the LiAc/SS-DNA/PEG Method. Genetic Transformation Systems in Fungi, Volume 1. A. M.

547 van den Berg and K. Maruthachalam. Cham, Springer International Publishing: 177-186.

548 González, A. and M. N. Hall (2017). "Nutrient sensing and TOR signaling in yeast and

549 mammals." The EMBO Journal.

550 Green, M. R. S., J. (2012). Protocol 6: Yeast Colony PCR.

551 Grossmann, G., J. Malinsky, W. Stahlschmidt, M. Loibl, I. Weig-Meckl, W. B. Frommer, M.

552 Opekarova and W. Tanner (2008). "Plasma membrane microdomains regulate turnover of

553 transport proteins in yeast." J Cell Biol 183(6): 1075-1088.

554 Grossmann, G., M. Opekarová, J. Malinsky, I. Weig-Meckl and W. Tanner (2007).

555 "Membrane potential governs lateral segregation of plasma membrane proteins and lipids

556 in yeast." The EMBO Journal 26(1): 1-8.

557 Helliwell, S. B., S. Losko and C. A. Kaiser (2001). "Components of a ubiquitin ligase complex

558 specify polyubiquitination and intracellular trafficking of the general amino acid permease."

559 The Journal of Cell Biology 153(4): 649-662.

560 Hinnen, A., J. B. Hicks and G. R. Fink (1978). "Transformation of yeast." Proceedings of the

561 National Academy of Sciences of the United States of America 75(4): 1929-1933.

562 lesmantavicius, V., B. T. Weinert and C. Choudhary (2014). "Convergence of ubiquitylation

563 and phosphorylation signaling in rapamycin-treated yeast cells." Mol Cell Proteomics 13(8):

564 1979-1992.

565 Kawai, S., W. Hashimoto and K. Murata (2010). "Transformation of Saccharomyces

566 cerevisiae and other fungi." Bioengineered Bugs 1(6): 395-403.

567 Kawai, S., T. A. Pham, H. T. Nguyen, H. Nankai, T. Utsumi, Y. Fukuda and K. Murata (2004).

568 "Molecular insights on DNA delivery into Saccharomyces cerevisiae." Biochem Biophys Res

569 Commun 317(1): 100-107.

570 Lauwers, E., G. Grossmann and B. Andre (2007). "Evidence for coupled biogenesis of yeast

571 Gap1 permease and sphingolipids: essential role in transport activity and normal control by

572 ubiquitination." Mol Biol Cell 18(8): 3068-3080.

573 Lin, C. H., J. A. MacGurn, T. Chu, C. J. Stefan and S. D. Emr (2008). "Arrestin-related

574 ubiquitin-ligase adaptors regulate endocytosis and protein turnover at the cell surface." Cell

575 135(4): 714-725.

576 Lin, C. H., J. A. MacGurn, T. Chu, C. J. Stefan and S. D. Emr (2008). "Arrestin-related

577 ubiquitin-ligase adaptors regulate endocytosis and protein turnover at the cell surface." Cell

578 135(4): 714-725.

579 Luo, G., A. Gruhler, Y. Liu, O. N. Jensen and R. C. Dickson (2008). "The sphingolipid long-

580 chain base-Pkh1/2-Ypk1/2 signaling pathway regulates eisosome assembly and turnover." J

581 Biol Chem 283(16): 10433-10444.

582 MacGurn, J. A., P. C. Hsu, M. B. Smolka and S. D. Emr (2011). "TORC1 regulates endocytosis

583 via Npr1-mediated phosphoinhibition of a ubiquitin ligase adaptor." Cell 147(5): 1104-1117.

584 Merhi, A. and B. Andre (2012). "Internal amino acids promote Gap1 permease ubiquitylation

585 via TORC1/Npr1/14-3-3-dependent control of the Bul arrestin-like adaptors." Mol Cell Biol

586 32(22): 4510-4522.

587 Mitrikeski, P. T. (2013). "Yeast competence for exogenous DNA uptake: towards

588 understanding its genetic component." Antonie van Leeuwenhoek 103(6): 1181-1207.

589 Moor, H. and K. Muhlethaler (1963). "Fine structure in frozen-etched yeast cells." J Cell Biol

590 17(3): 609-628. 
591

592

593

594

595

596

597

598

599

600

601

602

603

604

605

606

607

608

609

610

611

612

613

614

615

616

617

618

619

620

621

622

623

624

625

626

627

628

629

630

631

632

633

634

635

Moreira, K. E., S. Schuck, B. Schrul, F. Frohlich, J. B. Moseley, T. C. Walther and P. Walter (2012). "Seg1 controls eisosome assembly and shape." J Cell Biol 198(3): 405-420.

Murphy, E. R., J. Boxberger, R. Colvin, S. J. Lee, G. Zahn, F. Loor and K. Kim (2011). "Pil1, an eisosome organizer, plays an important role in the recruitment of synaptojanins and amphiphysins to facilitate receptor-mediated endocytosis in yeast." Eur J Cell Biol 90(10): 825-833.

Nikko, E. and H. R. Pelham (2009). "Arrestin-mediated endocytosis of yeast plasma membrane transporters." Traffic 10(12): 1856-1867.

Niles, B. J. and T. Powers (2014). "TOR complex 2-Ypk1 signaling regulates actin polarization via reactive oxygen species." Molecular Biology of the Cell 25(24): 3962-3972.

O'Donnell, A. F. (2012). "The running of the Buls: control of permease trafficking by $\alpha$ arrestins Bul1 and Bul2." Molecular and Cellular Biology 32(22): 4506-4509.

O'Donnell, A. F., A. Apffel, R. G. Gardner and M. S. Cyert (2010). "Alpha-arrestins Aly1 and Aly2 regulate intracellular trafficking in response to nutrient signaling." Mol Biol Cell 21(20): 3552-3566.

Opekarova, M., T. Caspari, B. Pinson, D. Brethes and W. Tanner (1998). "Post-translational fate of CAN1 permease of Saccharomyces cerevisiae." Yeast 14(3): 215-224.

Prosser, D. C., T. G. Drivas, L. Maldonado-Baez and B. Wendland (2011). "Existence of a novel clathrin-independent endocytic pathway in yeast that depends on Rho1 and formin." J Cell Biol 195(4): 657-671.

Prosser, D. C., A. E. Pannunzio, J. L. Brodsky, J. Thorner, B. Wendland and A. F. O'Donnell (2015). "alpha-Arrestins participate in cargo selection for both clathrin-independent and clathrin-mediated endocytosis." J Cell Sci 128(22): 4220-4234.

Seger, S., R. Rischatsch and P. Philippsen (2011). "Formation and stability of eisosomes in the filamentous fungus Ashbya gossypii." J Cell Sci 124(Pt 10): 1629-1634.

Stradalova, V., W. Stahlschmidt, G. Grossmann, M. Blazikova, R. Rachel, W. Tanner and J. Malinsky (2009). "Furrow-like invaginations of the yeast plasma membrane correspond to membrane compartment of Can1." J Cell Sci 122(Pt 16): 2887-2894.

Sun, Y., Y. Miao, Y. Yamane, C. Zhang, K. M. Shokat, H. Takematsu, Y. Kozutsumi and D. G. Drubin (2012). "Orm protein phosphoregulation mediates transient sphingolipid biosynthesis response to heat stress via the Pkh-Ypk and Cdc55-PP2A pathways." Mol Biol Cell 23(12): 2388-2398.

Toret, C. P. and D. G. Drubin (2006). "The budding yeast endocytic pathway." Journal of Cell Science 119(22): 4585-4587.

Vangelatos, I., K. Roumelioti, C. Gournas, T. Suarez, C. Scazzocchio and V. Sophianopoulou (2010). "Eisosome organization in the filamentous ascomycete Aspergillus nidulans." Eukaryotic Cell 9(10): 1441-1454.

Walther, T. C., J. H. Brickner, P. S. Aguilar, S. Bernales, C. Pantoja and P. Walter (2006). "Eisosomes mark static sites of endocytosis." Nature 439: 998.

Yashiroda, H., T. Oguchi, Y. Yasuda, E. A. Toh and Y. Kikuchi (1996). "Bul1, a new protein that binds to the Rsp5 ubiquitin ligase in Saccharomyces cerevisiae." Mol Cell Biol 16(7): 32553263.

Yu, S.-C., A. Dawson, A. C. Henderson, E. J. Lockyer, E. Read, G. Sritharan, M. Ryan, M. Sgroi, P. M. Ngou, R. Woodruff, R. Zhang, T. Ren Teen Chia, Y. Liu, Y. Xiang and P. D. Spanu (2016). "Nutrient supplements boost yeast transformation efficiency." Scientific Reports 6: 35738. 
636 Ziółkowska, N. E., R. Christiano and T. C. Walther (2012). "Organized living: formation

637 mechanisms and functions of plasma membrane domains in yeast." Trends in Cell Biology

638 22(3): 151-158.

639

640 Supplementary Table 1: Mutagenesis primers sequence

\begin{tabular}{|c|c|c|}
\hline & Forward & Reverse \\
\hline Tco89 & $\begin{array}{l}\text { ATGGTTCATCGAGGAAGGACTTTGAAGTCAGACACTGAT } \\
\text { GTAACATCTCTTAAGAAACCATTATTATCATGACA }\end{array}$ & $\begin{array}{l}\text { TCACCTTTGTTGTGGCTGTGTATGCATGTAGCCCACATTTTCCA } \\
\text { TCCTGC CCTGATGCGGTATTTTCTCCTTAC }\end{array}$ \\
\hline Art1 & $\begin{array}{l}\text { ATGGCATTTTCACGTCTTACATCTACTCATCAGTCCAATCA } \\
\text { TAACGGCTATAAGAAACCATTATTATCATGACA }\end{array}$ & $\begin{array}{l}\text { CTACTGGGTTATTCTATTGGAATCTAGAAAATCGGAAAAGTTT } \\
\text { TGTATTC CCTGATGCGGTATTTTCTCCTTAC }\end{array}$ \\
\hline Npr1 & $\begin{array}{l}\text { ATGTCTTCATTAACTCGATTGCTACAGGAAAAA } \\
\text { CGAAAAAATGAAACTTCTAAGAAACCATTATTA } \\
\text { TCATGACA }\end{array}$ & $\begin{array}{l}\text { TTATTGATTATTTTGCTTTTTTCTTTTTCTTTTCTAGGCCTGCAAT } \\
\text { ATGTGCCTGATGCGGTATTTTCTCCTTAC }\end{array}$ \\
\hline Bul1 & $\begin{array}{l}\text { ATGGCCAAAGATTTGAACGATTCGGGGTTTCCACCGAAG } \\
\text { AGGAAGCCTTTTAAGAAACCATTATTATCATGACA }\end{array}$ & $\begin{array}{l}\text { TTATTTTGTCACTTGCCTAACAGAAATAGGGATATCAATCTTC } \\
\text { GCTACGCCCTGATGCGGTATTTTCTCCTTAC }\end{array}$ \\
\hline Art3 & $\begin{array}{l}\text { ATGCCCATGGACCAATCTATCTCATCTCCATT } \\
\text { GTTTCCCATGGAAAAGGATAAGAAACCATTAT } \\
\text { TATCATGACA }\end{array}$ & $\begin{array}{l}\text { CTAAAGGGTACTCTCATTTATACTTTGTAATCCAG } \\
\text { ATTCATTATCTAACGCCTGATGCGGTATTTTCTCC } \\
\text { TTAC }\end{array}$ \\
\hline Seg1 & $\begin{array}{l}\text { ATGTTTAGAAGAAGAACAACTGCACCAGAAATGGAACAG } \\
\text { GCGGACCCGACTAAGAAACCATTATTATCATGACA }\end{array}$ & $\begin{array}{l}\text { CTATTTCTTTCTACCAAAGATTTTTTTTCAGTTTTTTTGCCGAAACT } \\
\text { ACCCTCCTGATGCGGTATTTTCTCCTTAC }\end{array}$ \\
\hline Ypk1 & $\begin{array}{l}\text { ATGTATTCTTGGAAGTCAAAGTTTAAGTTTGGAAAATCTA } \\
\text { AAGAAGAAAATAAGAAACCATTATTATCATGACA }\end{array}$ & $\begin{array}{l}\text { CTATCTAATGCTTCTACCTTGCACCATTGAGCTACCTAGCTGTT } \\
\text { CATTTCCCTGATGCGGTATTTTCTCCTTAC }\end{array}$ \\
\hline
\end{tabular}

641

642 Supplementary Table 2: Diagnostic primers sequence

\begin{tabular}{|l|l|l|}
\hline & Forward & Reverse \\
\hline $\begin{array}{l}\text { Tco89 } \\
\text { Inside/Outside }\end{array}$ & GGACGACCTGACTAGAGA & ACCATTTGTCTCCACACC \\
\hline $\begin{array}{l}\text { Tco89 } \\
\text { Outside/Outside }\end{array}$ & TAACCGCAAGGATAGCTAGTTGCG & GAGATACGGAATCCAGCGAAATCG \\
\hline $\begin{array}{l}\text { Art1 } \\
\text { Inside/Outside }\end{array}$ & AACCGTTGATGCTGATGAGGAG & TATGGTGCACTCTCAGTACAATCTGC \\
\hline $\begin{array}{l}\text { Art1 } \\
\text { Outside/Outside }\end{array}$ & TTCGGAGGAGAACGCTGTTG & ACGTGGCAGATTGTTGAAGATATACG \\
& & \\
\hline
\end{tabular}


bioRxiv preprint doi: https://doi.org/10.1101/244889; this version posted January 8, 2018. The copyright holder for this preprint (which was

not certified by peer review) is the author/funder, who has granted bioRxiv a license to display the preprint in perpetuity. It is made available under aCC-BY-NC-ND 4.0 International license.

Yu et al. 24

\begin{tabular}{|l|l|l|}
\hline $\begin{array}{l}\text { Npr1 } \\
\text { Inside/Outside }\end{array}$ & TATGGGCCGCCTTAGTTGTATAGAG & AGTTCCAATCCAAAAGTTCACCTGTC \\
\hline $\begin{array}{l}\text { Npr1 } \\
\text { Outside/Outside }\end{array}$ & TATGGGCCGCCTTTAGTTGTATAGAG & GCACTTTCTAAAGCTTCTTTGCTGTG \\
\hline $\begin{array}{l}\text { Bul1 } \\
\text { Inside/Outside }\end{array}$ & GTTCGTGTGTGTCAACAGGTATATCG & TAGATCGGCAAGTGCACAAACAATAC \\
\hline $\begin{array}{l}\text { Art3 } \\
\text { Inside/Outside }\end{array}$ & ACTCCCTTGCGCCATCTATC & GCATCCGCTTACAGACAAGC \\
\hline $\begin{array}{l}\text { Seg1 } \\
\text { Inside/Outside }\end{array}$ & TTTCAGTGCTCCCTTACTTTACCG & GCTTACATCAACACCAATAACGCC \\
\hline $\begin{array}{l}\text { Ypk1 } \\
\text { Inside/Outside }\end{array}$ & ATTACGAACATATCGAATGCGAGCAG & TAATAACAGACATACTCCAAGCTGCC \\
\hline
\end{tabular}

